University of Nebraska - Lincoln

DigitalCommons@University of Nebraska - Lincoln

January 2006

\title{
ANATOMY AND PHYSIOLOGY OF GIANT NEURONS IN THE ANTENNIFORM LEG OF THE AMBLYPYGID PHRYNUS MARGINEMACULATUS
}

\author{
A. J. Spence \\ University of California at Berkeley, Berkeley, California \\ Eileen Hebets \\ University of Nebraska - Lincoln, ehebets2@unl.edu
}

Follow this and additional works at: https://digitalcommons.unl.edu/bioscihebets

Part of the Behavior and Ethology Commons

Spence, A. J. and Hebets, Eileen, "ANATOMY AND PHYSIOLOGY OF GIANT NEURONS IN THE ANTENNIFORM LEG OF THE AMBLYPYGID PHRYNUS MARGINEMACULATUS" (2006). Eileen Hebets Publications. 6.

https://digitalcommons.unl.edu/bioscihebets/6

This Article is brought to you for free and open access by the Papers in the Biological Sciences at DigitalCommons@University of Nebraska - Lincoln. It has been accepted for inclusion in Eileen Hebets Publications by an authorized administrator of DigitalCommons@University of Nebraska - Lincoln. 


\title{
ANATOMY AND PHYSIOLOGY OF GIANT NEURONS IN THE ANTENNIFORM LEG OF THE AMBLYPYGID PHRYNUS MARGINEMACULATUS
}

\author{
A. J. Spence: Department of Integrative Biology, 3060 Valley Life Science Building \\ \#3140, University of California at Berkeley, Berkeley, California 94720-3140, USA. \\ E-mail: aspence@rvc.ac.uk
}

E. A. Hebets: School of Biological Sciences, 324 Manter Hall, University of Nebraska, Lincoln, Nebraska 68588, USA.

\begin{abstract}
Amblypygids have modified front legs that are not used for locomotion, but rather to probe the environment in the manner of antennae. These elongate, motile sense organs are referred to as antenniform legs. We have found remarkable replication in structure and function of giant neurons in the antenniform leg of the amblypygid Phrynus marginemaculatus C. L. Koch 1841 when compared with other amblypygids. These neurons have such large diameter axons (several $\mu \mathrm{m}$ ) that their action potentials can be recorded outside the cuticle. Their cell bodies are found in the periphery, in the distal-most segments of the antenniform leg, centimeters away from the central nervous system. Primary afferents from sense organs on the antenniform leg synapse onto some of the giant fibers in these distal segments of the leg. Standard histological techniques and a novel whole mount preparation were used to identify the location of giant cell bodies within the antenniform leg. We found several new cell bodies in segments 10-20, three of which were predicted by previous electrophysiological studies of another amblypygid, Heterophrynus elaphus Pocock 1903. Electrophysiology was used to show that the structure and function of four of the giant neurons, GN1, 2, 6, and 7, is very similar in P. marginemaculatus and H. elaphus. Heterophrynus elaphus inhabits humid tropical forests in South America while P. marginemaculatus individuals were collected from a pine rock hammock in the Florida Keys, USA. The similarity of findings in species with such distinct habitats suggests that the giant neurons are required for basic neuromechanical operation of these extended limbs, and are not subject to intense selection via ecological factors.
\end{abstract}

Keywords: Whip spider, giant neurons, mechanoreceptor, antennae

Giant neurons are a specialization of the nervous system in many animals that has enabled investigators to observe directly how neuron structure and function bring about behavior. Having exceptionally large diameter axons, giant neurons conduct action potentials very quickly (on the order of one to tens of meters per second). As conduction velocity scales with the square root of diameter, this increase in axon diameter and hence conduction velocity is one way in which organisms can reduce the conduction time of nervous signals. This results in faster turnaround from perceived stimuli to motor response. Selection pressure for fast escape behaviors is thought to have led to the development of the giant neurons found in squid, fish, crayfish, crickets, flies and cockroaches (Levine \& Tracey 1973; Tauber \& Camhi 1995; Mizrahi \& Libersat 1997; Eaton et al. 2001; Jablonski \& Straus- feld 2001). The function of the giant neurons in the above cases has been demonstrated clearly; their unusually rapid spike conduction time facilitates fast escape behavior.

Giant neurons and the conspicuous rapid behaviors they underlie have led to notable advancements in neuroscience. For example, in teleost fish, giant Mauthner neurons facilitate rapid evasive turning behavior. As a result, the entire neural pathway from sensory input (predator approach angle) to motor output (bilateral flexing of trunk muscles) is well understood and has been modeled mathematically (Eaton et al. 2001). Studies of windsensitive giant interneurons in the cricket are demonstrating how dendritic morphology produces complex spatiotemporal responses (Jacobs \& Theunissen 2000). Finally, the painted redstart (Myioborus pictus) employs a visual display specialized to trigger giant neuron me- 
diated escape responses, enabling them to forage more effectively (Jablonski \& Strausfeld 2001). In all of these examples, giant neurons have served as a model system for understanding the neural system in general, at the anatomical, physiological, and behavioral levels, and have offered insight into the neural basis of behavioral and ecological adaptations.

Amblypygids (Arachnida, Amblypygi), commonly called whip spiders, have a system of giant neurons in their first pair of legs that, in addition to having unique morphology and synaptic connectivity, has not yet been linked to any behavior. Unlike most other arachnids, these nocturnal predators do not use this first pair of legs for locomotion. The legs instead are elongated, motile, sensory appendages that are used to probe the environment in a manner similar to insect antennae. The antenniform legs are very long relative to body size. At approximately $5 \mathrm{~cm}$ long for an adult Phrynus marginemaculatus C. L. Koch 1841, the antenniform legs are 5 times the width of the prosoma and 2.5 times longer than the walking legs, yet are very thin, measuring only 150 $\mu \mathrm{m}$ in diameter at the distal end. They are used in orientation, prey capture, agonistic displays, and even courtship displays (Weygoldt 1972, 1974; Beck \& Gorke 1974; Foelix \& Hebets 2001; Fowler-Finn \& Hebets 2006). The tips of the antenniform legs are covered with various types of sensilla (Beck et al. 1974, 1977; Foelix et al. 1975; Igelmund 1987; Weygoldt 2000; Foelix \& Hebets 2001), some of which have been shown (using electrophysiology) to have mechanosensory (Igelmund \& Wendler 1991b) or olfactory (Hebets \& Chapman 2000) function. Several other types of sensilla are also found on the antenniform leg tip, some of unknown function, and others that are morphologically similar to contact chemoreceptive or hygroreceptive sensilla, though this latter function has not been demonstrated with electrophysiology (Foelix et al. 1975; Beck et al. 1977; Foelix \& Troyer 1980). The two nerves inside the antenniform leg contain some 20,000 small primary sensory axons (typically 100-200 $\mathrm{nm}$ in diameter) projecting from these sensilla, but they also contain several conspicuous giant neurons (axon diameter up to $12 \mu \mathrm{m}$ ).

Several features differentiate these giant neurons from those found in any other taxa. The cell bodies, or somata, of these giant neu- rons are found in the periphery, located in specific segments of the antenniform leg tarsus, in some cases centimeters away from the central nervous system (CNS). While some of these giant neurons are interneurons, others are proprioceptors. Synapses between primary afferent neurons and the giant interneurons also occur in the periphery, in the antenniform leg, a feature first discovered by Foelix (1975). Some of these synapses are axo-axonal, connecting the primary sensory neuron's axon to the axon of a giant interneuron. In almost all other arthropods studied to date, primary afferents project all the way into the CNS before synapsing onto second order neurons, making this peripheral integration a unique feature. Equally intriguing is the fact that the primary afferents, in addition to synapsing onto the giants, project in parallel all the way to the CNS. So the animal receives fast, highly summed information from the giant interneurons, and slower, sense organ specific sensory information in parallel. It is unknown whether primary afferents from olfactory or contact chemosensory sensilla synapse onto any of the giants, although stimulation with common odorants does not elicit spikes in the giants in another species of amblypygid, Heterophrynus elaphus Pocock 1903 (Igelmund \& Wendler 1991a, b).

As intriguing as the morphological differences are, even more mysterious is the behavioral role of the amblypygid giant neuron system. The system does not seem to underlie escape or foraging behaviors. Touching the antenniform leg in a manner sufficient to elicit spikes in the mechanosensitive giants does not elicit an escape response of the animal, or even a reliable retraction of the antenniform leg (Igelmund \& Wendler 1991b). In prey capture, touching a prey item with the antenniform leg does not directly precede the rapid strike movement, and often a delay of several seconds occurs between the touch and the strike (Foelix et al. 2002). Courtship and intraspecific aggressive behaviors are accompanied by high-speed $(\sim 30 \mathrm{~Hz})$ flicking of the antenniform leg; whether they are mediated by the giant neurons remains to be seen (Weygoldt 2002; Fowler-Finn \& Hebets 2006).

Although the majority of the 136 described species of amblypygid inhabit tropical and subtropical habitats, a few species are found in the temperate zones and still others inhabit 
arid and semi-arid regions (Weygoldt 2000). With such a range of habitat types, one might expect environmental factors to play an important role in shaping the sensory biology of ecologically disparate species. The purpose of this study was to characterize the giant neuron system of Phrynus marginemaculatus and compare it with previously studied amblypygids, in an attempt to shed light on the behavioral role of these giant fibers. The specific question that drove this study was whether these giant neurons are broadly used tools, supporting a variety of functions, or whether they are more specialized, facilitating speciesspecific tasks. Prior to this study, only the giant neurons in $H$. elaphus had been studied in detail using both histology and electrophysiology (Igelmund 1984, 1987; Igelmund \& Wendler 1991a, b). Our studies suggest that while there are some similarities and differences between species, the giant neurons appear to remain conserved across disparate ecological niches.

We chose to study $P$. marginemaculatus for several reasons. While it is in the same family as $H$. elaphus (Phrynidae), the species used for earlier neurophysiological work, the two genera do not appear closely related (Weygoldt 1996). P. marginemaculatus is also the species about which we know the most. It has been the subject of several behavioral studies including those focusing on life cycle and development (Weygoldt 1970), reproductive behavior (Weygoldt 1969, 1974), male-male contests, and female-female contests (Weygoldt 1969; Fowler-Finn \& Hebets 2006). Furthermore, the habitats of $H$. elaphus and $P$. marginemaculatus differ greatly. H. elaphus is found on the vertical surfaces of large buttressed trees underneath the dense tropical forest canopies of South America. Their habitat is extremely heterogeneous both in terms of physical structure and biotic composition. In contrast, $P$. marginemaculatus is found horizontally underneath limestone rocks in the relatively open pine rock hammocks of the Florida Keys, USA where the complexity of both physical and biotic structure is likely much lower. Comparing the giant neuron structure and function between $P$. marginemaculatus and the previously studied $H$. elaphus not only adds to our knowledge of the unique structure of amblypygid giant neurons, but also pro- vides insights in the behavioral role of this giant neuron system.

\section{METHODS}

Specimens.-Adult male and female whip spiders (Phrynus marginemaculatus) were collected from Big Pine Key, Florida (24.67N, $81.35 \mathrm{~W}$ ) on 6-9 November 2002 and were brought back to the laboratory where they were housed and cared for in an identical manner to a previous study (Hebets \& Chapman 2000). Voucher specimens are available from the personal collection of E. Hebets.

The antenniform leg of $P$. marginemaculatus comprises a femur ( $\sim 1 \mathrm{~cm}$ long), tibia ( $\sim 1.7 \mathrm{~cm}$ long), and tarsus ( $\sim 2 \mathrm{~cm}$ long), resulting in an appendage $\sim 5 \mathrm{~cm}$ in length (relative to a body length of $1 \mathrm{~cm}$ ). The tibia and tarsus are made up of many cylindrical smaller segments, called pseudosegments, giving rise to visible segmental boundaries and repeated sensory structures, and having length on the order of roughly $0.5-1 \mathrm{~mm}$. In keeping with past convention, the pseudosegments of the antenniform leg are labeled with increasing numbers starting at the distal-most tip. The tip segment then is 1 (segment is sometimes abbreviated $\mathrm{S}$, hence the tip segment is S1), moving proximally with increasing number to the most proximal segment of the tarsus, at the tarsus-tibia joint, which was typically segment 59 (S59).

Histology.- - Standard histological protocols were used to stain and image cross- and longitudinal sections of the antenniform leg. Three techniques were used. The first consisted of Propidium Iodide staining of whole mount preparations (Duch et al. 2000). The antenniform leg was clipped distal to the patella, and dissected in Schneider's culture medium at room temperature (RT). A sliver of razor blade was used to shave approximately the top third of the cuticle from the distalmost 30 segments of the tarsus. Tissue was fixed in $3.5 \%$ paraformaldehyde in PBS (phosphate buffered saline), and rinsed in several changes of PBS for $30 \mathrm{~min}$. In two preparations, the tissue was incubated in RNase A (Sigma, $0.1 \mathrm{mg} / \mathrm{ml}$, in PBS) for $30 \mathrm{~min}$ at $37^{\circ}$ $\mathrm{C}$, to reduce background staining. After being rinsed for 30 min in PBST (PBS + 0.3\% Triton-X 100), the tissue was incubated in Propidium Iodide (Sigma, 1:1000 in PBST) for $60 \mathrm{~min}$ at RT. After final rinses in PBS, the 
tissue was mounted on a slide with VectaShield mounting media (VectaLabs, Inc.).

Methylene blue and Osmium Tetroxide stains were utilized with resin (Ultra Low Viscosity Embedding Kit, Polysciences, Inc.) embedded tissue. For both, the antenniform leg was cut into short $(2-5 \mathrm{~mm})$ tubes and fixed with an aldehyde based fixative. Osmium Tetroxide staining proceeded as documented in Igelmund \& Wendler 1991a. Tissue was cut into 5,8 , and $10 \mu \mathrm{m}$ thick sections using a Leitz 1512 microtome. Tissue that had not been stained with $\mathrm{OsO}_{4}$ was stained on the slide with $1 \%$ methylene blue solution, before being mounted with Entellan mounting media. Slides were imaged with a Leica DM4000B compound microscope and DFC480 digital camera.

Propidium iodide staining of whole mount preparations provided adequate contrast to image the large cell bodies, was the most rapid imaging technique, and had the advantage of leaving long intact lengths of leg, which made noting in which segment each cell body was found an easy task. Due to the need to conserve our limited number of specimens for the electrophysiological studies, we discontinued our histological studies upon obtaining images adequate to resolve the axons within the nerve cross section and the locations of giant cell bodies.

Electrophysiology.-Extracellular recordings from the antenniform leg were made using a technique similar to that of Igelmund \& Wendler (1991a). Animals were anesthetized with $\mathrm{CO}_{2}$ or by placing them on a bed of ice for $3 \mathrm{~min}$. Once anesthetized, they were restrained using strips of dental wax, and covered with a moist Kim-wipe to prevent desiccation. The antenniform leg was extended laterally and woven through four pairs of metal pins. These were made by cutting one side of a 16-pin DIP socket into four small plastic pieces, each containing two pins spaced by $2.54 \mathrm{~mm}$. Electrochemical connection between each pin and the leg was made using a small amount of EEG paste. These pins constituted four pairs of differential recording electrodes, with the spacing between each pair being approximately $5 \mathrm{~mm}$, that were connected to the positive and negative inputs of a differential voltage amplifier. As the distalmost 50 segments of the tarsus that we were interested in typically measured between 1 and $1.5 \mathrm{~cm}$, we placed the electrodes as close together as possible. The amplifier was a custom built, miniature 16 channel extracellular voltage amplifier, with a gain of 1000 . The 4 amplified signals were digitized $(20 \mathrm{kHz}$ sample rate) by a 16 channel FireWire A/D box (DAQPad, National Instruments, Inc.), and acquired directly into MATLAB (Mathworks, Inc.) for analysis.

Multichannel spike waveforms were analyzed in MATLAB using custom scripts that performed an amalgam of the most popular manual and automated sorting techniques (Lewicki 1998; Spence et al. 2003). Briefly, the raw waveforms were bandpass filtered (passband: $300 \mathrm{~Hz}$ to $5 \mathrm{kHz}$ ), occurrences of spikes were detected and sorted by amplitude using an energy window filter, and then the distributions of propagation times between channels were used to identify unique spike types. The cleanest (i.e., not overlapping with other spikes on any other channel) individual spikes were extracted from the raw recordings, aligned on channel four, and averaged to produce a spike template (for details, see Spence et al. 2003). Each of these "templates" for a particular multichannel spike having distinct spike amplitude on each channel and distinct propagation time between channels is assumed to originate from an individual giant fiber.

Stimuli were applied manually under a dissecting microscope. For mechanosensory stimuli, a small plastic rod was used to gently deflect bristle hairs at various points along the antenniform leg. Two bouts of $30 \mathrm{sec}$ of stimulation were applied at each point. For deflection stimuli, the same rod was used to deflect the antenniform leg laterally in the plane of the animal.

\section{RESULTS}

External morphology.- - Ten molted, preserved, or whole mount antenniform legs were qualitatively surveyed with an optical microscope for the presence and distribution of sensilla. Examination confirmed the presence of bristle, club, porous and rod hair sensilla in addition to modified tarsal claws, a pit organ, and a plate organ (Igelmund 1987). The leaflike hairs reported on $H$. elaphus were not systematically found, with a single leaf-like hair being found on only one animal out of $10 \mathrm{ob}-$ served. The rod sensilla are grouped in a sin- 
gle oval shaped patch on segment 1 . The plate organ was typically found on segment 11 , once on segment 13, and in one case two plate organs appeared: one each on segments 11 and 13. A bulbous (and angled relative to the plane perpendicular to the leg axis) segmental boundary appears at the S22/S23 boundary, as opposed to the $\mathrm{S} 21 / 22$ boundary in $H$. elaphus. This boundary likely contains the large slit sensilla reported for the similar boundary in $H$. elaphus, but we were unable to resolve the slit in the optical microscope.

Histology.-The internal morphology of the antenniform leg of $P$. marginemaculatus is similar to that of $H$. elaphus. At segment 40 , the antenniform leg is approximately 140 $\mu \mathrm{m}$ in diameter (Figs. 1-3). Visible within the tarsus are 2 tendons, the lumen, a blood vessel and 2 large nerves. The nerves contain several large axons, the most readily apparent of which are 2 giant axons located in nerve 1 , and 5 others in nerve 2 (Figs. 2, 3). These are in addition to an estimated 20,000-30,000 primary sensory afferents of much smaller diameter, of order $0.1 \mu \mathrm{m}$, contained in fascicles (Foelix \& Troyer 1980). In this segment (40), the largest seven axons have effective radii $(=$ $\left.\mathrm{r}_{\text {eff }}\right)$ of $1.8,2.1,2.3,2.5,2.6,5.1$, and $6.8 \mu \mathrm{m}$ respectively, where the effective radius is computed from

$$
r_{e f f}=\sqrt{\frac{\mathrm{A}}{\pi}}
$$

and " $\mathrm{A}$ " is the measured cross-sectional area of the axon $(n=1)$.

Longitudinal sections of the antenniform leg revealed giant cell bodies in segments 5 , $6,10,11,12,14,15,16,20,22,23$, and 26 (Figs. 4-10). These somata are several tens of $\mu \mathrm{m}$ in length and width (the largest was in segment 6 , measuring $\sim 100$ by $50 \mu \mathrm{m}$, Fig. 4 ), and are readily identified by their large size and distinct structure. They consist of an oval shaped outer cell body, a circular, outlined nucleus with homogenous, lightly stained interior, and an innermost nucleolus, which appears as a spot. The Propidium iodide stained cell bodies (Figs. 4-7) display a white outlined nucleus with dark interior, and the nucleolus appears as a white spot. The Osmium Tetroxide (Fig. 4) and Methylene Blue (Figs. $7,10)$ stained cell bodies have the reverse contrast. It is assumed that these cell bodies give rise to the giant axons seen in Figs. 1-3.
The cell bodies in segments 11 and 26 were of slightly smaller size, having narrower and more elongated cell bodies, but still displaying the distinct circular nucleus and nucleolus (Figures 4-10). Finding the location of the cell bodies within the antenniform leg is useful because these results can be compared with multi-site electrophysiological recordings, establishing a connection between the observed cytology and sensory physiology.

Electrophysiology.-Large $(\sim 50 \mu \mathrm{V}-1.5$ $\mathrm{mV}$ ) action potentials of varied amplitude and conduction velocity were recorded across 4 positions on the antenniform leg tarsus (Fig. 11). The spacing between pins in an individual recording pair was $2.54 \mathrm{~mm}$, and the spacing between pairs was approximately $5 \mathrm{~mm}$ (see Fig. 11). For the purposes of understanding spike timing and conduction velocity, the spike can be thought of as "at" the midpoint between a pair of recording sites at the time when the inner part of its spike waveform crosses zero. Spontaneous activity from smaller units was common, while mechanical stimulation (brushing hairs or bending the leg) was required to elicit bursts of spikes from larger units. At least 7 distinct types of spikes were observed. Four of these were elicited repeatedly and classified reproducibly ( $n=5$ different animals) using mechanosensory stimulation. Stimulation of the bristle hairs at 3 different points along the tarsus (Fig. 11) was adequate to identify spikes corresponding to two of the giant neurons, which we label GN1 and GN2 following the convention of Igelmund \& Wendler (1991a).

The largest spike, corresponding to GN1, had peak-to-peak amplitude $1.3 \mathrm{mV}$ at segment 52 (recording site 4), and an average conduction velocity of $2.9 \mathrm{~m} / \mathrm{s}$. It responded to deflections of bristle hairs, maximally at the tarsus tip and with reduced sensitivity as the stimulation site was moved proximally (Fig. 11). The GN1 spike is generated at the site of stimulation, and propagates both proximally and distally inside the neuron, appearing on our most distal recording site at segment 10 . GN1 is most likely the largest axon (Figs. 13 , Axon 1), and one of the cell bodies in segment 5 or 6 (Fig. 4), due to its large spike amplitude and appearance on our segment 10 recording site.

The same bristle hair stimulation traces were also adequate to identify spikes origi- 


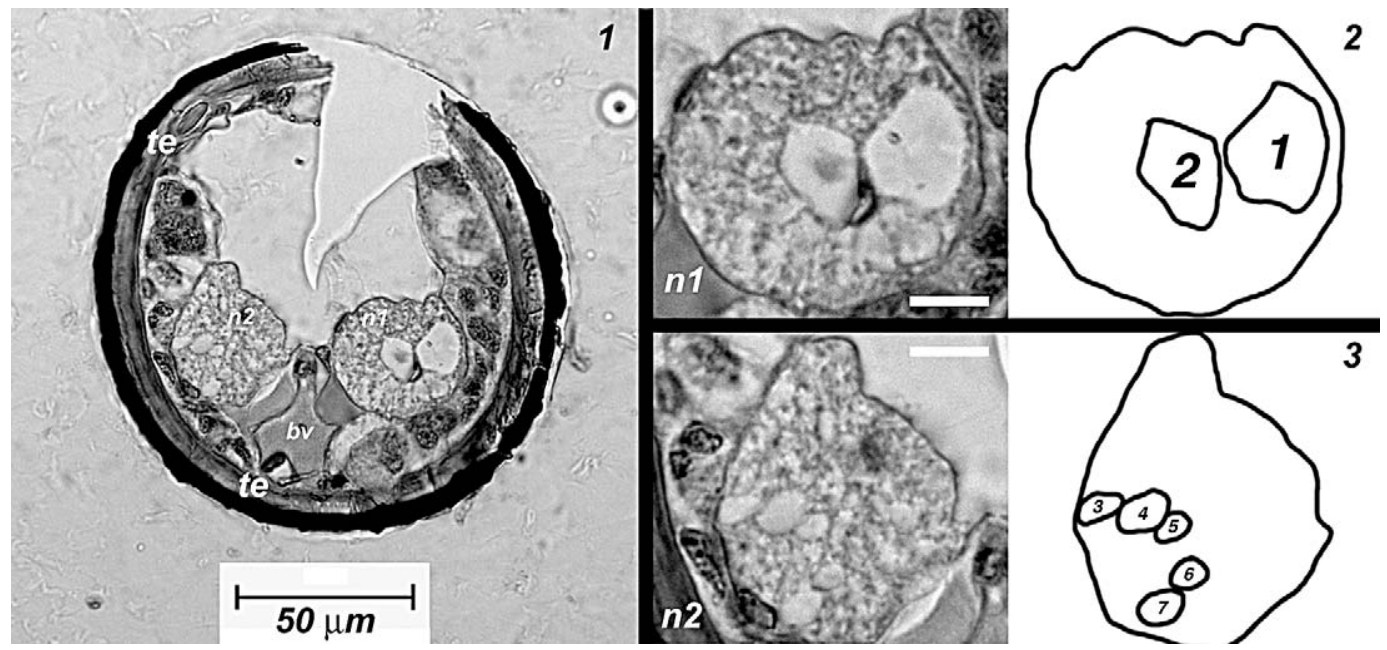

Figures 1-3. Figure 1.-Cross section of the tarsus of the antenniform leg, stained with methylene blue. Labeled are two tendons (te), a blood vessel (bv), and the two nerves (n1 and n2). Enlarged views of nerves $\mathrm{n} 1$ and $\mathrm{n} 2$ are seen at right (Figures 2,3), with outlines of the nerve bundle and largest seven giant axons. Scale bars in Figures 2 and 3 are $10 \mu \mathrm{m}$.

nating from GN2. Although this neuron's spike also originates at the point of stimulation and propagates in both directions, it is smaller in amplitude and conduction velocity than GN1 (0.41 $\mathrm{mV}$ and $2.6 \mathrm{~m} / \mathrm{s})$, and does not appear on our segment 10 recording site (Fig. 11). This places its cell body between segments 10 and 28. These traits suggest that it is GN2, and based on the similarity of our results to those of Igelmund \& Wendler (1991a), it seems likely that it consists of one of the axons of intermediate size (Figs. 1-3, Axons 2-7), and one of the cell bodies found in segment 23 (Figs. 9, 10). The amplitudes and conduction velocities of both GN1 and GN2 we have found are similar to those found in $H$. elaphus (Igelmund 1984). The firing rates of GN1 and GN2 adapted quickly, with repeated stimulation of the same bristle hairs producing few spikes.

Two types of spike that responded to deflection of the antenniform leg near segment 20 were identified. Deflection (bending) of the tarsus at other points produced fewer or no spikes from these neurons. We did not localize the exact segmental boundary for which bending maximally elicits these spikes. The fact that these spikes responded to bending of the tarsus, propagated solely proximally, and did not appear on our segment 12 recording site (suggesting the cell body is proximal to seg- ment 12 but distal to segment 28) identifies them as GN6 and 7. These spikes had relatively small average amplitude and conduction velocity (GN6: $0.20 \mathrm{mV}$ and $1.8 \mathrm{~m} / \mathrm{s}$, GN7: $0.16 \mathrm{mV}$ and $1.7 \mathrm{~m} / \mathrm{s}$ ). The amplitude of these spikes is in good agreement with that found for them in $H$. elaphus, which varied between 0.1 and $0.2 \mathrm{mV}$ (Igelmund 1984).

\section{DISCUSSION}

Morphology of the antenniform leg of $\boldsymbol{P}$. marginemaculatus. - We found that $P$. marginemaculatus has a close replication of the sensory physiology found in $H$. elaphus. The tarsus of the antenniform leg is equipped with similar classes of sensory organs, and with similar distribution. The rod hairs are grouped in a single oval-shaped patch on the first tarsal segment, which is similar to $H$. elaphus, but in contrast to $H$. longicornis Butler 1873, in which they are grouped in 3 distinct circular patches on each of the first 3 segments (Igelmund 1987). We did not repeatedly find the leaf-like hairs found on specific segments of $H$. elaphus (Igelmund 1987). In this manner, $P$. marginemaculatus is similar to $H$. longicornis and $H$. batesii Butler 1873, which also lack the leaf-like hairs (Igelmund 1987).

Internally, the antenniform leg of $P$. marginemaculatus has a neural architecture that closely parallels previously studied amblypy- 


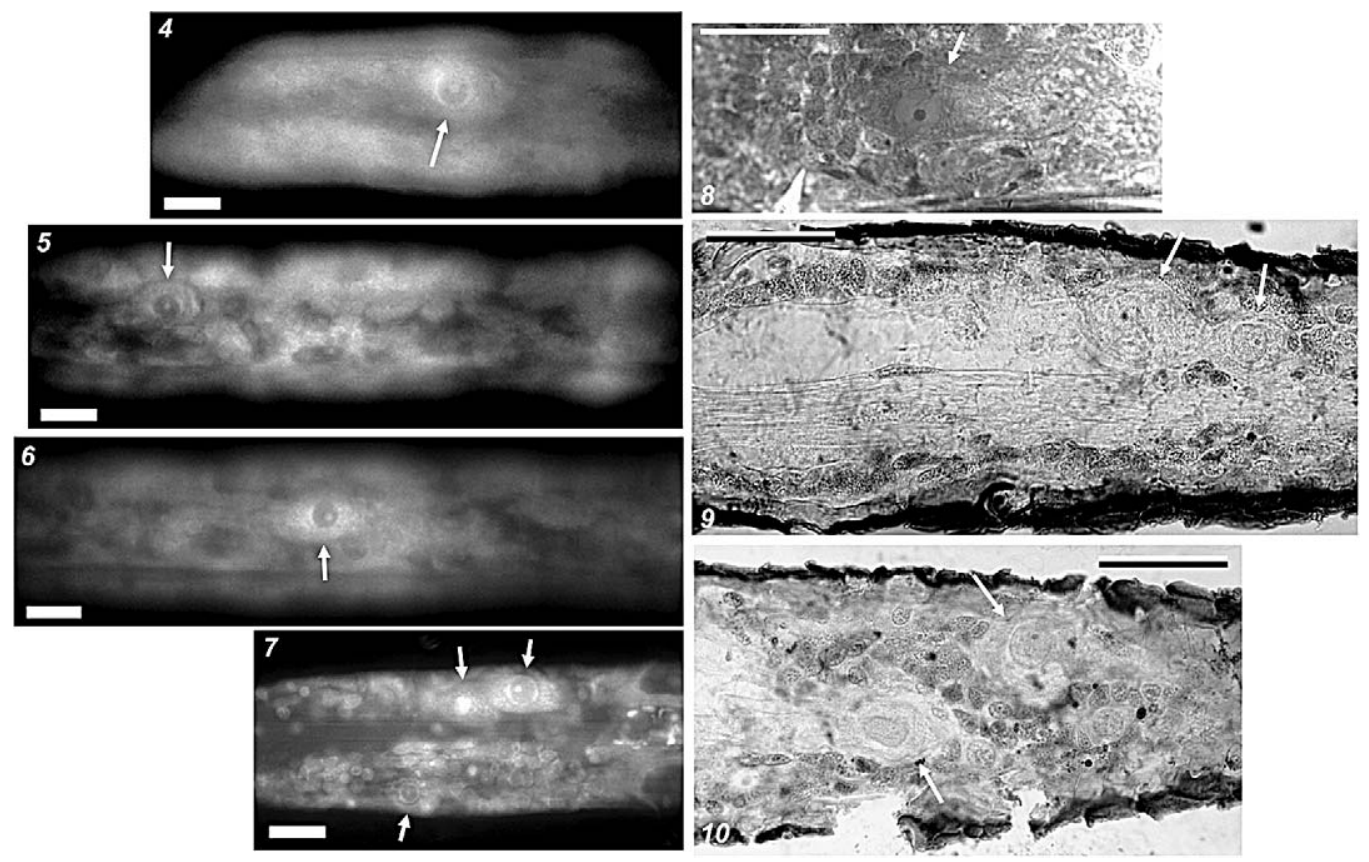

Figures 4-10.- Representative longitudinal sections of the antenniform leg. Whole mount Propidium iodide stains of the following segments: (Figure 4) 6, (Figure 5) 10, (Figure 6) 12, and (Figure 7) 22, respectively. Osmium tetroxide stain of segment 10 (Figure 8, different specimen from Figure 5), and methylene blue stained sections of segment 23 (Figures 9 and 10). Single cell bodies (arrows) are visible in segments 6 (Figure 4), 10 (Figures 5 and 8), and 12 (Figure 6). Three are seen in segment 22 (Figure 7), and 23 (Figures 9 and 10; these are serial sections through the same tissue; two cell bodies are seen in Figure 9, and a third appears in Figure 10). Cell bodies (cf. panel Figure 4) consist of outer cell body membrane (in this case $\sim 100 \mu \mathrm{m}$ wide by $50 \mu \mathrm{m}$ tall), inner nucleus (white circular line enclosing dark area, here $26 \mu \mathrm{m}$ diameter) and innermost nucleolus (inner white spot, $6 \mu \mathrm{m}$ diameter). Scale bars $=$ $50 \mu \mathrm{m}$.

gids. The giant axons in $P$. marginemaculatus are distributed between the two nerves with remarkable similarity to $H$. elaphus. The largest 2 axons, presumably GN1 and GN2, are situated adjacent to each other in one nerve, while the remaining smaller axons are bundled together in the other nerve. The peripheral giant cell bodies and sensory synapses found in whip spiders (Amblypygi), whip scorpions (Uropygi), and harvestmen (Opiliones) are rare, and to date this type of neural architecture has only been found in a few cases within the animal kingdom (Foelix 1975; Foelix \& Troyer 1980). Insects and other arthropods are thought to have their neuronal cell bodies and the first site of synaptic integration located centrally, either in the brain or ganglia.

We used a new preparation and staining method to image the giant cell bodies: whole mount dissection of the antenniform leg followed by Propidium Iodide labeling (Figs. 47). We found that the unique cytology of the giant neuron somata, especially the homogeneous, light staining inside the nucleus but outside the nucleolus, was reproduced with the Propidium Iodide stain (Figs. 4-7). This peculiar staining and large nucleus size led previous investigators to ask whether these cells were polyploid, but a Feulgen stain established that this was unlikely (Foelix \& Troyer 1980). This new preparation provided a more rapid technique to locate in which segment each cell body lies, and resulted in longer, multi-segment pieces of intact antenniform leg for observation. This more readily enables observation of structures that span multiple segments, such as nerves, tendons, and blood vessels.

Giant cell bodies in segments 10-20, and 


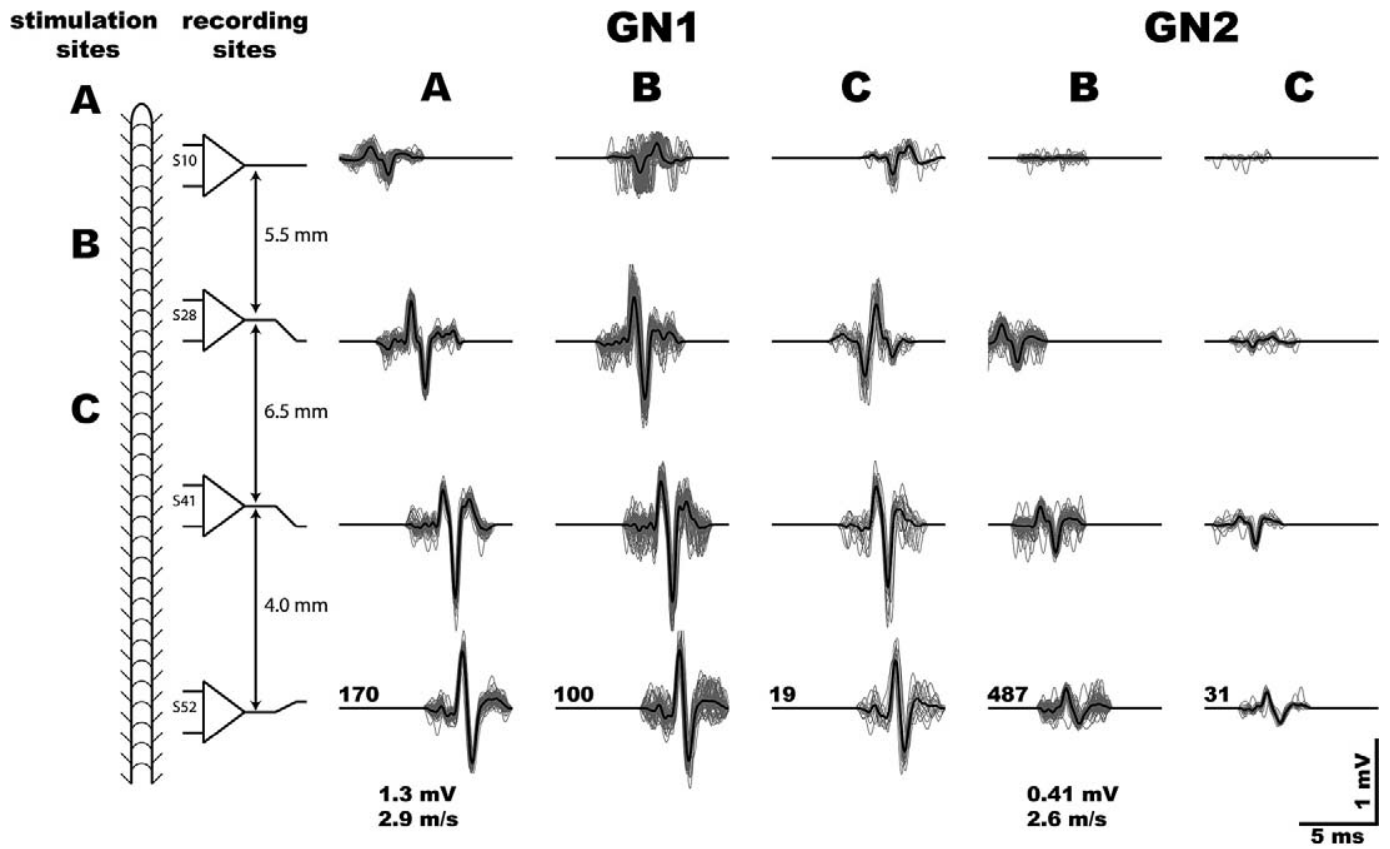

Figure 11.-Four point recording from the tarsus, and spikes from GN1 and GN2. Average spike (dark line) overlaid on individual spikes (gray lines). The number of individual spikes averaged to calculate each template is shown on the fourth recording site waveform. Peak-to-peak amplitude of average waveform on fourth site, and average conduction velocity are shown below the first GN1 and GN2 spike types. Propagation direction can be seen in time course between channels and as a reversal of peak order (proximal $=$ positive then negative, distal $=$ negative then positive). Spikes were aligned in time on the fourth recording site, and thus the variation in conduction time is most easily seen in the "jitter" of spikes on channel 1. The geometry of the recording pins is not drawn to scale. Separation between pins within a recording site is $2.54 \mathrm{~mm}$, and the center to center spacing between pairs was approximately $5 \mathrm{~mm}$. As a result the spacing between pins on the edge of neighboring pairs was also approximately $2.5 \mathrm{~mm}$. The measured center to center spacings for this recording are indicated with arrows.

GN3, 4 and 5.- In P. marginemaculatus we have found giant cell bodies in segments 5,6 , $10,11,12,14,15,16,20,22,23$, and 26. In H. longicornis, H. batesii, and H. elaphus giant cell bodies were found most frequently in segments $1,5,6,13,19,20,21,22,25$ and 101 (Foelix \& Troyer 1980; Igelmund \& Wendler 1991a; Foelix \& Hebets 2001). There is remarkable replication in the location of the cell bodies, with those in S5 and 6 being in the identical segment, and those in the region of 19-26 likely to be slightly shifted but homologous cell bodies. We found several new giant cell bodies between segments 10 and 20 . This finding agrees with the electrophysiological results of Igelmund \& Wendler (1991a), whose recordings predicted that the cell bodies of GN3, 4 and 5 would lie in this region. Now that the segmental location of these giant neuron cell bodies is known, tracer-fills of sensory neurons on individual segments can be pursued to look for connectivity between the giant neurons and the various sensilla. In addition to bristles, these sensilla include the club and porous hairs that are found on the distalmost $\sim 20$ segments, which are thought to have hygrometric and olfactory function, respectively. This is in addition to the slit sense organs that are found on each tarsal segment, which may sense cuticular stress or segmental deflection.

The sensory function of GN3, 4, and 5 is unknown. Although we recorded additional types of spikes that matched examples from these neurons in $H$. elaphus (Igelmund \& Wendler 1991a), we could not elicit their activity with basic mechanosensory or odor stimuli. The odor stimuli used were a leaf- 


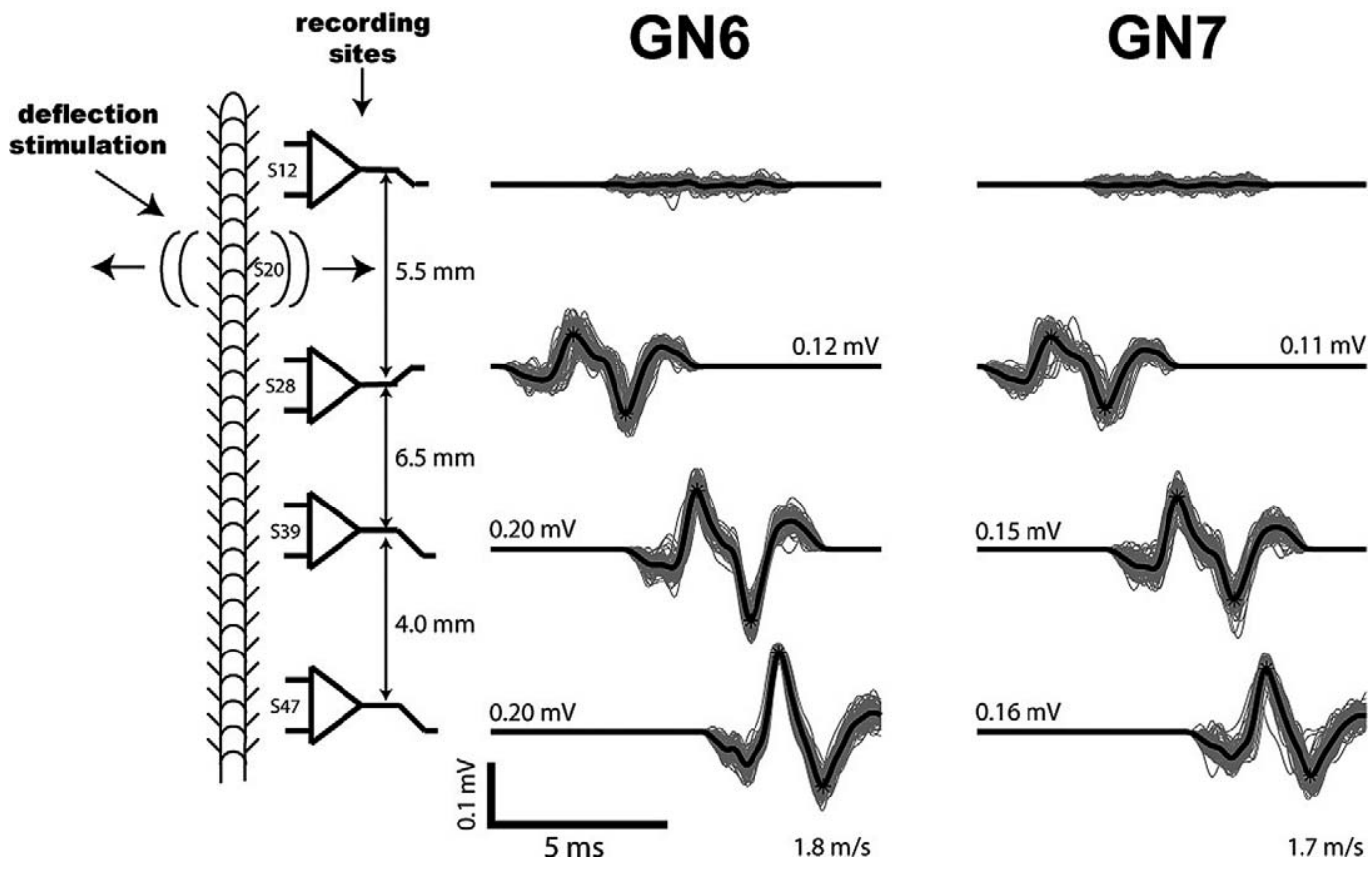

Figure 12.-Four point recording from the tarsus, and spikes from GN6 and GN7. Average peak to peak amplitudes are shown for each recording site, and the average conduction velocity denoted below. Both templates are averages of 100 individual spikes.

alcohol and a leaf-aldehyde, odorants commonly emitted by plants. This confirms some of the results for $H$. elaphus, in which mechanical, olfactory, and even temperature and hygrometric stimuli did not elicit a response from these neurons, apart from a phasic response of GN5 to tobacco smoke (Igelmund \& Wendler 1991a).

Structure and function of giant neurons 1, 2, 6 and 7.- GN1 and GN2 are mechanosensory interneurons that respond to deflections of the bristle hairs. We were able to identify them clearly in $P$. marginemaculatus using several factors: their spike amplitude and conduction velocity, response to bristle hair stimulation, spike initiation at the point of stimulation and propagation in both directions, and the location of their cell bodies implied by the recording sites (i.e. GN1 distal to segment 10, GN2 between segments 10 and 28). We qualitatively tested the receptive fields of GN1 and GN2 in P. marginemaculatus and found agreement with $H$. elaphus: stimulation at the tip of the antenniform leg produces only GN1 spikes, stimulation at segment 20 produces both GN1 and GN2 spikes, and nearing segment 45 only GN2 spikes are elicited. Thus it appears $P$. marginemaculatus has a similar organization of GN1 and 2: GN1 covers the distalmost 20 segments, ramping down its sensitivity moving proximally as GN2 begins to take over, becoming more sensitive as segment 40 is approached. GN1 and 2 adapt quickly to repeated stimulation of the same bristles. Given their large (several $\mathrm{mm}$ ) receptive fields and rapid adaptation, GN1 and 2 appear to function as rapid touch detectors for the tarsus.

GN6 and 7 are sensory neurons. They function as rapid proprioceptors, giving the animal feedback in the amount and direction of bending at the segment $22 / 23$ joint. We were able to identify GN6 and 7 in P. marginemaculatus using similar criteria: their smaller amplitude spikes always originated at the same point (between segments 12 and 28), propagated solely proximally, and responded to deflection of the leg, all of which matches the behavior previously found in $H$. elaphus. GN6 and 7 are thought to be either coupled to a large slit sense organ at the segment $22 / 23$ border, or part of a separate joint receptor mechanism at 
that boundary. Either connectivity could allow them to perform their observed proprioceptive function.

Comparing histology with multi-site electrophysiology, it was found that in H. elaphus the GN1 cell body is the one found in segment 5, GN2 is in segment 23, and GN6 and 7 are two of four found in segment 22. Our electrophysiological results suggest that $P$. marginemaculatus has markedly similar structure and function of GN1, 2, 6, and 7. GN1 is one of the cell bodies in segment 5 or 6 , GN2 is most likely in segment 23, and GN6 and 7 are two of those found in segment 22.

The role of the giant neurons in natural behavior.- The role of the giant fiber system of amblypygids in the natural behavior of the animal remains a mystery. Although there are cases in which giant fibers are not directly linked to specific behaviors (DiCaprio 2003), typically, in other arthropods, giant neurons facilitate rapid escape or predatory behavior (Levine \& Tracey 1973; Tauber \& Camhi 1995; Mizrahi \& Libersat 1997). Touching the antenniform leg in a manner that elicits spikes in GN1 or 2, even repeatedly, does not evoke a rapid escape response of the amblypygid, while puffs of air directed at trichobothria on the walking legs usually does. Giant neurons can underlie rapid predatory behaviors (Gronenberg 1995a, b), but while amblypygids make rapid prey strikes with their pedipalps, the antenniform legs do not touch the prey immediately before a strike, and often a period of seconds will elapse between the last touch of the antenniform leg and the strike (pers. obs.).

Rapid tapping and vibratory movements are made with the antenniform legs during courtship and intraspecific aggressive behaviors (Weygoldt 2000; Fowler-Finn \& Hebets 2006). High speed video of aggressive behaviors (Fowler-Finn \& Hebets 2006) has found the frequency of tapping to be $\sim 30 \mathrm{~Hz}$. Spikes from GN1 take on the order of $30 \mathrm{~ms}$ to get to the CNS, and so it is possible that the animal could use GN1 to receive feedback during each cycle of the tapping behavior. While feedback at the same rate as the tapping may not be needed to regulate the behavior, it would be required in order to react to changes within a single cycle. Spikes in the primary afferents could not provide feedback on the time scale of a single tapping cycle: applying local circuit theory to these unmyelinated axons, we predict conduction velocity to scale as the square root of axon radius (Aidley 1998), and so estimate that primary afferents having a radius 20 times smaller than the giants would take $\sim 140 \mathrm{~ms}$ to arrive at the CNS. This would give a maximum feedback driven tapping rate of about $7 \mathrm{~Hz}$. However, the rapid adaptation of GN1 to stimulation of the same bristles, and the lack of an obvious need for feedback, makes this hypothesis (that GN1 exists to provide fast feedback for rapid vibrations) unlikely. It is possible that some of the other motor or proprioceptive giants facilitate this high-speed tapping of the antenniform legs. As the vibratory tapping occurs during courtship and aggressive behaviors, it seems likely that it signals individual quality, and is used as a basis of assessment of a mate or competitor. Whether information about quality is contained in the frequency of the vibration or some other component of the signal remains open. If the frequency of antenniform leg vibration were to signal the quality of an individual, however, this could be a source of evolutionary pressure on the development of a faster sensorimotor system in the antenniform leg.

Amblypygids will intermittently exhibit rapid retraction of the antenniform leg when touched, a behavior that appears highly dependent on the animal's state of alertness (Spence pers. obs.). Given the costs of losing these appendages (animals missing both legs cannot orient or proactively hunt), and perhaps even the costs of being entrapped by them, fast touch detection and rapid proprioceptive feedback may simply be required for adequate maneuverability in such long appendages.

The sense organs and underlying giant fiber system we have studied in P. marginemaculatus is remarkably similar to that of $H$. elaphus, yet the habitats of these two species, the Florida Keys for $P$. marginemaculatus and Brazilian rainforest for $H$. elaphus, are quite different. One predicts that the environment of $P$. marginemaculatus would offer a smaller diversity of prey, fewer vertical surfaces, more seasonality, a lack of canopy and correspondingly more light, and lower humidity than the Brazilian home of $H$. elaphus. The similarity in the giant neuron systems across these species suggest that they are crucial for the mo- 
tility and basic function of the antenniform legs, and as such are not under great selection pressure from these ecological differences.

\section{ACKNOWLEDGMENTS}

The authors would like to thank all the members of the Hebets laboratory, including Ginevra Ryman, Jennifer Wesson, Nicole VanderSal, and Kasey Fowler-Finn. Migumi Fuse, Simon Sponberg, Susan Somerville, and members of the UC Berkeley Arachnology Discussion group provided valuable critical feedback. We would also like to acknowledge the assistance of the Hoy Laboratory, especially Dr. Patricia Rivlin, Prof. Elke Buschbeck, Damien Elias, and Prof. Ron Hoy. We would also like to thank the United States Department of the Interior Fish and Wildlife Service at National Key Deer Refuge for a special use permit. The experiments in this study fully comply with the NIH guidelines for animal care, publication No. 86-23, revised 1985, and with all state and federal laws.

\section{LITERATURE CITED}

Aidley, D.J. 1998. The Physiology of Excitable Cells. Fourth edition. Cambridge University Press, New York. 489 pp.

Beck, L. \& K. Gorke. 1974. Tagesperiodik, Revierverhalten und Beutefang der Geißelspinne Admetus pumilio C. L. Koch. Zeitschrift für Tierpsychologie 35:173-186.

Beck, L., R. Foelix, E. Godeke \& R. Kaiser. 1974. Über die Haarsensillen der Geißelspinne Admetus pumilio (Arach., Amblypygi). Naturwissenschaften 61:327-328.

Beck, L., R. Foelix, E. Goedeke \& R. Kaiser. 1977. Morphologie, Larvantwicklung und Haarsensillen des Tastbeinpaares der Geißelspinne Heterophrynus longicornis Butler (Arach., Amblypygi). Zoomorphologie 88:259-276.

DiCaprio, R.A. 2003. Nonspiking and spiking proprioceptors in the crab: nonlinear analysis of nonspiking TCMRO afferents. Journal of Neurophysiology 89:1826-1836.

Duch, C., R.J. Bayline \& R.B. Levine. 2000. Postembryonic development of the dorsal longitudinal flight muscle and its innervation in Manduca sexta. Journal of Comparative Neurology 422:117.

Eaton, R.C., R.K.K. Lee \& M.B. Foreman. 2001. The Mauthner cell and other identified neurons of the brainstem escape network of fish. Progress in Neurobiology 63:467-485.

Foelix, R.F. 1975. Occurrence of synapses in peripheral sensory nerves of arachnids. Nature 254: 146-148.
Foelix, R.F., I.W. Chu-Wang \& L. Beck. 1975. Fine structure of tarsal sensory organs in the whip spider Admetus pumilio (Arachnida, Amblypygi). Tissue \& Cell 7:331-346.

Foelix, R.F. \& E.A. Hebets. 2001. Sensory biology of whip spiders (Arachnida, Amblypygi). Andrias 15:129-140.

Foelix, R.F. \& D. Troyer. 1980. Giant neurons and associated synapses in the peripheral nervous system of whip spiders. Journal of Neurocytology 9:517-536.

Foelix, R.F., D. Troyer \& P. Igelmund. 2002. Peripheral synapses and giant neurons in whip spiders. Microscopy Research and Technique 58: 272-282.

Fowler-Finn, K.D. \& E.A. Hebets. 2006. An examination of agonistic interactions in the whip spider Phrynus marginemaculatus (Arachnida, Amblypygi). Journal of Arachnology 34:62-76.

Gronenberg, W. 1995a. The fast mandible strike in the trap-jaw ant Odontomachus. 1. Temporal properties and morphological characteristics. Journal of Comparative Physiology A: Neuroethology, Sensory, Neural, and Behavioral Physiology 176:391-398.

Gronenberg, W. 1995b. The fast mandible strike in the trap-jaw ant Odontomachus. 2. Motor control. Journal of Comparative Physiology A 176: 399-408.

Hebets, E.A. \& R.F. Chapman. 2000. Electrophysiological studies of olfaction in the whip spider Phrynus parvulus (Arachnida, Amblypygi). Journal of Insect Physiology 46:1441-1448.

Igelmund, P. 1984. Elektrophysiologische und morphologische Untersuchungen zur Funktion peripherer Riesenneurone in den Tastbeinen der Geisselspinne Heterophrynus elaphus Pocock. Doctoral thesis. Cologne University, Cologne, Germany.

Igelmund, P. 1987. Morphology, sense-organs, and regeneration of the forelegs (whips) of the whip spider Heterophrynus elaphus (Arachnida, Amblypygi). Journal of Morphology 193:75-89.

Igelmund, P. \& G. Wendler. 1991a. The giant fiber system in the forelegs (whips) of the whip spider Heterophrynus elaphus Pocock (Arachnida, Amblypygi). Journal of Comparative Physiology A: Neuroethology, Sensory, Neural, and Behavioral Physiology 168:63-73.

Igelmund, P. \& G. Wendler. 1991b. Morphology and physiology of peripheral giant interneurons in the forelegs (whips) of the whip spider Heterophrynus elaphus Pocock (Arachnida, Amblypygi). Journal of Comparative Physiology A: Neuroethology, Sensory, Neural, and Behavioral Physiology 168:75-83.

Jablonski, P.G. \& N.J. Strausfeld. 2001. Exploitation of an ancient escape circuit by an avian predator: relationships between taxon-specific 
prey escape circuits and the sensitivity to visual cues from the predator. Brain Behavior and Evolution 58:218-240.

Jacobs, G.A. \& F.E. Theunissen. 2000. Extraction of sensory parameters from a neural map by primary sensory interneurons. Journal of Neuroscience 20:2934-2943.

Levine, J. \& D. Tracey. 1973. Structure and function of giant motorneuron of Drosophila melanogaster. Journal of Comparative Physiology 87: 213-235.

Lewicki, M.S. 1998. A review of methods for spike sorting: the detection and classification of neural action potentials. Network: Computation in Neural Systems 9:R53-R78.

Mizrahi, A. \& F. Libersat. 1997. Independent coding of wind direction in cockroach giant interneurons. Journal of Neurophysiology 78:26552661.

Spence, A.J., R.R. Hoy \& M.S. Isaacson. 2003. A micromachined silicon multielectrode for multiunit recording. Journal of Neuroscience Methods 126:119-126.

Tauber, E. \& J. Camhi. 1995. The wind-evoked escape behavior of the cricket Gryllus bimaculatus: integration of behavioral elements. Journal of Experimental Biology 198:1895-1907.

Weygoldt, P. 1969. Beobachtungen zur Fortpflanzungsbiologie und zum Verhalten der Geißelspinne Tarantula marginemaculata C. L.
Koch (Chelicerata, Amblypygi). Zeitschrift für Morphologie der Tiere 64:338-360.

Weygoldt, P. 1970. Lebenszyklus und postembryonale Entwicklung der Geißelspinne Tarantula marginemaculata C.L. Koch (Chelicerata, Amblypygi) in Laboratorium. Zeitschrift für Morphologie der Tiere 67:58-85.

Weygoldt, P. 1972. Geisselskorpione und Geisselspinne (Uropygi und Amplypygi). Zeitschrift des Koelner Zoo 15:95-107.

Weygoldt, P. 1974. Kampf und Paarung bei der Geisselspinne Charinus montanus Weygoldt. Zeitschrift für Tierpsychologie 34:217-223.

Weygoldt, P. 1996. Evolutionary morphology of whip spiders: towards a phylogenetic system (Chelicerata: Arachnida: Amblypygi). Journal of Zoological Systematics and Evolutionary Research 34:185-202.

Weygoldt, P. 2000. Whip Spiders (Chelicerata: Amblypygi): Their Biology, Morphology and Systematics. Apollo Books, Stenstrup, Denmark. $163 \mathrm{pp}$.

Weygoldt, P. 2002. Fighting, courtship, and spermatophore morphology of the whip spider $M u$ sicodamon atlanteus Fage, 1939 (Phrynichidae) (Chelicerata, Amblypygi). Zoologischer Anzeiger 241:245-254.

Manuscript received 12 May 2005, revised 30 May 2005. 